\title{
Characteristics of gas-forming pyogenic liver abscess caused by Klebsiella pneumoniae and Clostridi perfringens
}

\section{Zheng-Yang Chen}

306 hospital of PLA

Nan Jiang

306 hospital of PLA

Song Guo

306 hospital of PLA

Bin-Bin Li

306 hospital of PLA

Jia-Qi Yang

306 hospital of PLA

Shao-Bin Chai

306 hospital of PLA

Hong-Feng Yan

306 hospital of PLA

He-Ming Yang

306 hospital of PLA

Jin-Lian Zhou

306 hospital of PLA

Hong-Wei Sun

306 hospital of PLA

Yan Cui ( $\nabla$ dryancui@163.com )

306 hospital of PLA https://orcid.org/0000-0002-6833-5611

\section{Research article}

Keywords: Clostridium perfringens; Diabetes mellitus; Gas-forming pyogenic liver abscess; Klebsiella pneumonia

Posted Date: September 23rd, 2019

DOI: https://doi.org/10.21203/rs.2.14789/v1 
License: (c) (i) This work is licensed under a Creative Commons Attribution 4.0 International License. Read Full License 


\section{Abstract}

Background: Gas-forming pyogenic liver abscess is a life-threatening disease with poor prognosis commonly caused by 2 bacteria, Klebsiella pneumoniae and Clostridium perfringens. Due to its low incidence and associated high mortality rate, it is important to study the biological characteristics of the disease. The aim of this study was to conduct a worldwide review of literature on gas-forming pyogenic liver abscess caused by K. pneumoniae and C. perfringens. Methods: We searched PubMed and Web of Science databases from January 2009 to March 2019, with published in English. All relevant articles were accessed in full text. The manual search included references of retrieved articles. Finally, 35 publications were selected for review. Results: The results showed that more cases of gas-forming pyogenic liver abscess in Asia were caused by K. pneumoniae than by $C$. perfringens $(P=0.011)$. The prevalence of diabetes mellitus in patients with gas-forming pyogenic liver abscess caused by K. pneumoniae was higher than caused by $C$. perfringens $(P=0.032)$. The survival rate of patients with gas-forming pyogenic

liver abscess caused by K. pneumoniae who received surgical debridement or drainage was higher than caused by $C$. perfringens $(P=0.002)$. Conclusions: The prevalence of diabetes mellitus was higher in patients with gas-forming pyogenic liver abscess caused by $\mathrm{K}$. pneumoniae than in patients caused by $\mathrm{C}$. perfringens .

\section{Background}

Gas-forming pyogenic liver abscess (GFPLA) was first described by Smith and accounts for $7 \%$ to $24 \%$ of pyogenic liver abscesses (PLAs) [1-3]. Gas-forming abscess is a classical feature of this infection and may facilitate early recognition and treatment. Diabetes mellitus (DM) is a well-known risk factor of PLAs and may play a role in gas formation in PLAs [3]. In recent times, the incidence of GFPLA has been increasing gradually due to advancements in medical technology in the area of liver diseases, such as hepatocellular carcinoma (HCC), with the development of radiofrequency ablation and transarterial chemoembolization [4-5].

Klebsiella pneumoniae and Clostridium perfringens are the 2 bacteria that most commonly cause GFPLA. The clinical manifestations of GFPLA caused by $K$. pneumoniae (GFPLA-Kp) and $C$. perfringens (GFPLA$C p$ ), which include fever and right upper quadrant abdominal pain, are not different from those of nonGFPLA [6]. However, there are some clinical differences. Earlier studies reported a statistically higher incidence of septic shock, multiple organ dysfunction syndrome, bacteremia, and mortality in patients with GFPLA [7-8]. The variety in clinical characteristics may be due to the different virulence factors. The $\mathrm{K} 1$ and $\mathrm{K} 2$ capsular serotypes of $K$. pneumoniae, with a prevalence of $52 \%$ to $77.6 \%$ in PLAs caused by $K$. pneumoniae, were considered risk factors for liver abscess [9]. Studies have shown that these 2 serotypes play a key role in impairing neutrophil phagocytosis in patients with $D M$, which leads to severe infection with gas formation in the liver [10]. In GFPLA- $C p$, alpha-toxin is the major virulence factor and can trigger hemolysis, which progresses rapidly, and acidosis and renal failure develop swiftly [11-12]. 
Most reports of GFPLA are from Asia, with Taiwan as an example [7]. The organisms most commonly associated with GFPLA are K. pneumoniae and C. perfringens [13]. K. pneumoniae, a gram-negative bacteria, can be found in the upper respiratory and intestinal tracts of humans and can cause a wide variety of infectious diseases, such as urinary tract infections and respiratory tract infections [14]. GFPLA$K p$ is 1 such disease discussed here. In contrast, $C$. perfringens is an anaerobic gram-positive rod-shaped bacteria that produces spores. It is in the normal flora of the gastrointestinal and genitourinary tracts of healthy humans. Under certain disease conditions, such as DM and malignant cancers, $C$. perfringens can become pathogenic and cause bloodstream and gas-forming infections that can lead to significant mortality [15].

The incidences of GFPLA-Kp and GFPLA- $C p$ have recently been increasing from year to year. However, there is a lack of systematic and comprehensive evaluation of GFPLA-Kp and GFPLA- $C p$ in the past several years. Hence, relevant literature published from January 2009 to March 2019 were searched to compare clinical features.

\section{Methods}

The PubMed database was searched using the following terms: "gas forming" AND "Liver abscess" AND "K. pneumoniae," "gas forming" AND "Liver abscess, pyogenic" AND "K. pneumoniae," "gas" AND "Liver abscess" AND "K. pneumoniae," "gas forming" AND "Liver abscess, pyogenic" AND "K. pneumoniae," "gas forming" AND "Liver abscess" AND "C. perfringens," "gas forming" AND "Liver abscess, pyogenic" AND "C. perfringens," "gas" AND "Liver abscess" AND "C. perfringens," "gas forming" AND "Liver abscess, pyogenic" AND "C. perfringens." Next, full publications (not abstracts) from the Web of Science database using the same terms were searched to supplement the research. The references of the articles were then searched to make sure no eligible studies were missed. The search was restricted to papers published from January 2009 to February 2019. At the end, a total of 35 publications were included in this review.

Statistical analysis was performed using SPSS software, version 21.0 (SPSS Inc., Chicago, IL, USA). Data are expressed as mean \pm standard deviation. Inter-group comparisons were made using the Student's $t$ test and qualitative data were analyzed using the $\chi 2$ test. Statistical differences were considered

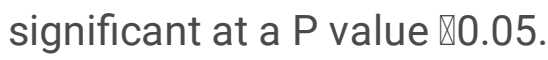

\section{Results}

Table $1[11-12,16-37]$ and Table $2[8,38-47]$ show the clinical characteristics and laboratory test results of patients with GFPLA-Kp (12 cases) and patients with GFPLA-Cp (24 cases). The mean ages of patients 
with GFPLA- $K p$ and patients with GFPLA-Cp were $67.08 \pm 10.22$ years and $66.70 \pm 8.78$ years, respectively. The proportions of patients with GFPLA-Kp and patients with GFPLA-Cp of male sex were $58.3 \%$ and $70.8 \%$, respectively; however, there was no statistically significant difference between the 2 patient groups. It was also found that age $>70$ years was not a predictor of mortality in patients with GFPLA- $K p$ and patients with GFPLA- $C p$.

Of the 12 patients with GFPLA-Kp, 11 (91.7\%) were from Asia (4 from Japan, 3 from Taiwan, 2 from Korea, 1 from China, and 1 from Australia) and 1 (8.3\%) was from the USA. In contrast, of the 24 patients with GFPLA-Cp, 11 (45.8\%) were from Asia (6 from Japan, 1 from China, 2 from Hong Kong, and 2 from Australia), 9 (37.5\%) were from Europe (2 from the UK, 2 from Spain, 2 from Germany, 2 from the Netherlands, and 1 from Belgium), and 4 (16.6\%) were from the USA. With regard to the patients from Asia, the proportion of patients with GFPLA-Kp was greater than the proportion of patients with GFPLA-Cp $(P=0.011)$.

Although Thng et al. reported that $K$. pneumoniae was the most common bacteria causing GFPLA, it is not clear what the most common bacteria causing GFPLA in patients with DM is [48]. Data showed that the most common underlying disease in patients with GFPLA-Kp was DM (83.3\%). Of the 24 patients with GFPLA- $C p, 10$ (41.6\%) had DM, 6 (25\%) had malignant tumors (1 patient with pancreatic cancer, 2 with gastric cancer, 2 with colon cancer, and 1 with HCC), 3 (12.5\%) had cholangitis, $3(12.5 \%)$ were previously in good health, 1 (4\%) had undergone a hemi-hepatectomy for liver metastasis of a neuroendocrine tumor with an unknown primary site, and 1 (4.1\%) had received a liver transplant for alcoholic cirrhosis. DM was more prevalent in patients with GFPLA- $K p$ than in patients with GFPLA- $C p(P=0.032)$. The results showed that DM is strongly associated with GFPLA-Kp. All but 3 patients (91.3\%) presented with fever and 2 patients (8.3\%) had no localizing signs.

Positive results of blood and pus cultures, blood culture, and pus culture were obtained in 6 patients (50\%), 5 patients (41.6\%), and 1 patient (8.3\%) with GFPLA-Kp, respectively. Furthermore, positive results of blood culture, pus culture, and blood and pus cultures were obtained in 19 patients (79.1\%), 3 patients $(12.5 \%)$, and 2 patients (8.3\%) with GFPLA- $C p$, respectively. The proportion of positive blood culture results was higher in patients with GFPLA- $C p$ than in patients with GFPLA-Kp (P=0.03) compared to the proportion of positive blood and pus culture results. However, there were no statistically significant differences in the results of blood and pus cultures, blood cultures, or pus cultures between patients with GFPLA- $K p$ and patients with GFPLA- $C p$. 
With regard to laboratory investigations (Table 3 [11-12, 16-37] and Table 4 [8, 38-47]), patients with GFPLA- $C p$ had higher levels of aspartate aminotransferase (AST) (869.20 $\pm 583.02 \mathrm{IU} / \mathrm{L}$ versus $233.12 \pm$ $98.19 \mathrm{IU} / \mathrm{L}, \mathrm{P}=0.007)$ and alanine aminotransferase (ALT) $(609.00 \pm 516.26 \mathrm{IU} / \mathrm{L}$ versus $199.85 \pm 74.93$ IU/L, $P=0.034$ ) than patients with GFPLA-Kp at hospital admission. However, no statistically significant differences were found in alkaline phosphatase levels between the 2 patient groups. The hemoglobin, bilirubin, and lactate dehydrogenase (LDH) levels in patients with GFPLA-Cp were $6.88 \pm 5.26 \mathrm{~g} / \mathrm{dL}, 6.30 \pm$ $7.08 \mathrm{mg} / \mathrm{dL}$, and $1867.74 \pm 2813.59 \mathrm{IU} / \mathrm{L}$, respectively. These laboratory index indicated a high incidence of hemolysis.

Because ultrasonography cannot reliably depict the detailed internal structure of the gas-containing lesions, the X-Ray, CT and MRCP findings were assessed in GFPLA-Cp and GFPLA- Kp. With regard to radiological investigations, all patients (except 1 unmentioned case) with GFPLA-Cp and all patients with GFPLA- $K p$ were diagnosed using computed tomography (CT), as it is the most reliable technology for diagnosing GFPLA caused by these organisms. Similarly, Lee et al. reported a $100 \%$ detection rate with CT [2]. Abdominal palpation and ultrasonography were less reliable for diagnosis. The changes on CT between the patient groups were similar and include the presence of low attenuation areas and cavity formation with or without gas in the liver. In contrast, these same changes were not well defined on abdominal palpation and ultrasound in the patient groups.

All 12 patients with GFPLA-Kp had solitary abscesses. Of the 24 patients with GFPLA-Cp (with the exception of 2 patients that were not specified), 21 patients (95.4\%) had solitary abscesses and 1 patient (4.5\%) had multiple abscesses. The abscesses were larger in patients with GFPLA-Cp than in patients with GFPLA-Kp $(15.98 \pm 21.93 \mathrm{~cm}$ versus $8.16 \pm 4.44 \mathrm{~cm})$; however, the difference in abscess size between the patient groups was not statistically significant. Abscesses of diameter $₫ 5 \mathrm{~cm}$ were found in $83.3 \%$ of patients with GFPLA-Kp and in $62.5 \%$ of patients with GFPLA-Cp. The location of abscesses (right, left, and both lobes) was compared using the $\chi 2$ test and no statistically significant differences were observed.

The treatment of GFPLA-Kp and GFPLA-Cp include antibiotic therapy, surgical debridement and drainage. For GFPLA-Kp and GFPLA-Cp, it is important to treat antibiotics before drug sensitivity test. However, we found that surgical debridement or drainage was performed in all 12 patients (100\%) with GFPLA-Kp and in 5 patients (20.8\%; 3 DM and 2 non-DM) with GFPLA-Cp. In other words, more patients with GFPLA-Kp received surgical debridement or drainage than patients with GFPLA-Cp $(P<0.001)$. The survival rate of patients with GFPLA-Kp who received surgical debridement or drainage was higher than that of patients with GFPLA-Cp who received the same treatment $(P=0.002)$. 
Considering the outcomes of GFPLA-Kp and GFPLA-Cp, twelve patients (50\%) with GFPLA-Cp died as a result of different complications. This mortality rate is higher than that of patients with GFPLA-Kp (16.7\%). However, no statistically significant differences in mortality rate between the patient groups were found using the $\chi 2$ test. There were also no statistically significant differences in the rates of death within 24 hours of hospital admission between DM and non-DM patients with GFPLA-Cp. Other cases have improved or even cured.

\section{Discussion}

GFPLA was first introduced by Smith in 1944 and several sporadic cases have been reported in many parts of the world since then. However, there are obvious regional differences in the incidence of GFPLA; for example, the incidence of GFPLA is low in the West but high in Asia, especially in Taiwan [2]. Our results were similar to those in earlier reports in that we also found that in Asia, the incidence of GFPLA$K p(91.7 \%)$ is higher than that of GFPLA- $C p(45.8 \%)(P=0.011)$. Although several hypotheses have been proposed, it is still unclear why GFPLA-Kp is more prevalent in Taiwan. One hypothesis stated that the prevalence can be explained by the spread of a pathogenic clone [49]. GFPLA has recently emerged as an important infectious disease in Japan, Korea, China, and Australia [8, 42, 50-51]. However, the correlation of these pathogenic clones to different regions using molecular typing methods is yet unclear. It was therefore concluded that this may also explain the high incidence of GFPLA-Kp in in other parts of Asia besides Taiwan.

It was found that age $>70$ years was not a predictor of mortality in patients with GFPLA-Kp and patients with GFPLA-Cp. This finding was contradicted by a multicenter observational study by Sartelli et al., who found that age $>70$ years can predict mortality [52]. The limited sample size of patients with GFPLA-Kp and patients with GFPLA-Cp may explain the contradiction.

With regard to the underlying diseases in patients with these 2 categories of GFPLA, we found that DM was more prevalent in patients with GFPLA- $K p$ than in patients with GFPLA- $C p(P=0.032)$. This is similar to the results of studies by Chou et al. and Yang et al. [6,53]. The higher incidence of GFPLA in patients with DM is because poor glycemic control leads to compromised immunity, neutrophil dysfunction, and chemotaxis dysfunction. These lead to poor microcirculation in the liver, with rapid growth and vigorous metabolism of bacteria in the affected areas, which may in turn lead to gas production [53]. Furthermore, poor glycemic control in patients with DM may provide gas-forming microorganisms with a more favorable environment for gas formation through mixed acid fermentation of glucose. Mixed acid fermentation within the abscess contributes to gas production resulting from carbon dioxide produced during glucose fermentation in liver tissues under anaerobic conditions. K. pneumoniae was reported to 
produce formic hydrogenlyase, an enzyme that is only produced in acidic environments when local $\mathrm{pH}$ reaches 6 or less by acid accumulation. Fermentation by formic hydrogenlyase is a key process that often leads to accumulation of acids, and formic acid accumulated within the abscess is converted to carbon dioxide and hydrogen gas by formic hydrogenlyase when the $\mathrm{pH}$ is 6 or less [2]. Poor glycemic control compromises the immune system and increases glucose metabolism via the polyol pathway. This depletes the nicotinamide adenine dinucleotide phosphate hydrogen necessary for superoxide production in neutrophil-mediated opsonophagocytosis [54], thereby providing a favorable microenvironment for the rapid growth and vigorous metabolism of the microorganisms and leads to gas formation ${ }^{[3]}$. However, an earlier study found that high glucose levels reflecting poor glycemic control may stimulate capsular polysaccharide (CPS) biosynthesis and CPS gene expression of highly virulent $K$. pneumoniae through reduction of cyclic adenosine monophosphate levels, which increases resistance to phagocytosis and contributes to the development of liver abscesses [55]. The CPS of $K$. pneumoniae is an acidic polysaccharide composed of 4-6 sugars, which seem to correlate with bacterial virulence [56]. These sugars play significant roles in K. pneumoniae infection and are explained below. (1) resistance to phagocytosis caused by CPS [57]: Poor glycemic control in patients with DM can increase CPS production. The increased CPS can block binding sites and resist the effects of conditioning and phagocytosis by macrophages, neutrophils, epithelial cells, and dendritic cells. (2) CPS inhibits the maturation of dendritic cells and reduces the production of interleukin-12 and tumor necrosis factor-alpha (TNF-a), leading to dysfunction of immature dendritic cells and ultimately limiting the migration of natural killer cells. This helps bacteria avoid attack from the host immune system and favors bacterial reproduction in the early stage of infection [58]. However, the details need to be further explored. The above-stated actions of CPS facilitate gas formation in patients with PLA caused by K. pneumoniae who have DM.

There are 5 serotypes of $C$. perfringens $(A, B, C, D$, and $E$ ) based on the production of 4 different lethal toxins (alpha, beta, epsilon, and iota) [59]. All 5 serotypes of $C$. perfringens produce alpha-toxin, which is a phospholipase $C$ that hydrolyzes lecithin to form phosphorylcholine and diglyceride [60]. It has been suggested that poor glycemic control may inhibit the action of phospholipase $C$ and limit the damage to alpha-toxin. This may explain why DM is less prevalent in patients with GFPLA-Cp than in patients with GFPLA- $K p$. However, the incidence of hemolysis in patients with GFPLA- $C p$ was higher than that in patients with GFPLA-Kp. Alpha-toxin is the main cause of hemolysis as it can hydrolyze the phospholipids in red blood cell membranes [60]. The $\mathrm{N}$-terminal and $\mathrm{C}$-terminal domains are structures of the alpha-toxin that form a loop. The former has phospholipase activity while the latter is hydrophobic and enters the cell membrane [60]. The loop formed by the C- and N-terminals contains GM1 gangliosidebinding motifs and specifically binds to GM1a. The alpha-toxin binding to GM1a triggers specific signaling events that lead to activation of tyrosine kinase $A$ and the subsequent signaling cascade results in the release of TNF-a, which causes catastrophic hemolysis and inflammation [61]. 
For the early diagnosis of $C$. perfringens infection, gram staining of the blood sample is important because it is a gram-positive rod, while $K$. pneumoniae is gram-negative. That is why there were more positive blood culture results in patients with GFPLA- $C p$ than in patients with GFPLA-Kp $(P=0.03)$. Therefore, for early diagnosis of GFPLA- $C p$, blood culture tests should be considered at hospital admission. In contrast, early diagnosis of GFPLA-Kp usually involves physical and laboratory examinations and CT scans.

Our review also revealed that GFPLA-Cp is associated with higher AST and ALT levels than GFPLA-Kp $(P<0.05)$. Furthermore, hemoglobin, $L D H$, and total or indirect bilirubin levels were found to be elevated in patients with GFPLA-Cp but not in patients with GFPLA-Kp. AST, ALT, LDH, and total or indirect bilirubin levels are indicators of liver function that may increase due to hemolysis caused by $C$. perfringens. This may explain the higher levels of AST and ALT observed in patients with GFPLA-Cp. However, most of the studies we reviewed were retrospective and it cannot be conclusively stated that the elevation in AST and ALT levels was due to hemolysis caused by $C$. perfringens.

It was found that a greater proportion of patients with GFPLA-Kp was treated with surgical debridement or drainage than that of patients with GFPLA-Cp $(P<0.01)$. It was suggested that this may be because of the higher prevalence of DM in patients with GFPLA-Kp. Concomitant DM and GFPLA- $K p$ can potentially lead to poor patient outcomes even within 1 or 2 days of hospitalization [62]. Furthermore, only 6 patients (3 DM and 3 non-DM) with GFPLA-Cp received surgical debridement or drainage and there was no statistically significant difference between them. This finding shows that greater attention should be paid to hemolysis and necessary measures should be taken. It also shows that surgical debridement or drainage was not necessary.

There were no statistically significant differences in gender between survivors and the deceased in patients with GFPLA- $C p$. However, an earlier study found that significantly more female than male patients survived $(P<0.05)$ [24]. The difference may be associated with basic patient conditions, such as the presence of underlying diseases, age, and nationality. We also found that a greater proportion of patients with GFPLA-Kp who received surgical debridement or drainage survived than that of patients with GFPLA- $C p(P=0.002)$. Patients with GFPLA often present with septic shock and bacteremia, which increase mortality [48]. Therefore, surgical debridement or drainage is vital.

\section{Conclusions}

In conclusion, based on the study characteristics, GFPLA may be considered a life-threatening disease with a wide spectrum. Although this review was limited by the small number of publications, we reckon 
that it will raise awareness for GFPLA-Kp and GFPLA-Cp.

\section{Declarations}

Ethics approval and consent to participate

None.

Consent for publication

Not applicable.

\section{Availability of data and materials}

The datasets used and/or analysed during themanuscript are available from the database of Pubmed and Web of Science.

\section{Competing interests}

The authors declare that they have no competing interests.

\section{Fundings}

No fundings support this work.

Author contributions: Cui Y designed the research; Jiang N, Guo S, and Lin BB Chai SB performed the research; Yan HF, Yang JQ, Sun HW, Yang HM contributed analytic tools; Zhou JL analyzed the data; Chen ZY wrote the paper.

\section{Acknowledgements}

The authors acknowledge all those who participated in the preparation of this manuscript. 


\section{Authors Information}

${ }^{1}$ Department of General Surgery, 306 Hospital of PLA, Beijing, Anxiang Beili outside Deshengmen, Chaoyang District, Beijing 100101, People's Republic of China. ${ }^{2}$ Department of General Surgery, the 306th Hospital of PLA-Peking University Teaching Hospital, Beijing, Anxiang Beili outside Deshengmen, Chaoyang District, Beijing 100101, People's Republic of China. ${ }^{3}$ Department of General Surgery, PLA 306 Clinical Hospital of Anhui Medical University, Beijing Anxiang Beili outside Deshengmen, Chaoyang District, Beijing 100101, People's Republic of China. ${ }^{4}$ Department of Pathology, 306 Hospital of PLA, Beijing, Anxiang Beili outside Deshengmen, Chaoyang District, Beijing 100101, People's Republic of China.

\section{References}

1. Smith RS. Pyogenic liver abscess in the aged. Am J Surg. 1944; 63:206-213.

2. Safe IP, Couceiro Kdo N, Martins AG. Gas-forming pyogenic liver abscess. Rev Soc Bras Med Trop. 2013;46:528.

3. Lee HL, Lee HC, Guo HR, Ko WC, Chen KW. Clinical significance and mechanism of gas formation of pyogenic liver abscess due to Klebsiella pneumoniae. J Clin Microbiol. 2004;42:2783-2785.

4. Shiina S, Tateishi R, Arano T, et al. Radiofrequency ablation for hepatocellular carcinoma: 10-year outcome and prognostic factors. Am J Gastroenterol. 2012;107:569-577.

5. Huang SF, Ko CW, Chang CS, Chen GH. Liver abscess formation after transarterial chemoembolization for malignant hepatic tumor. Hepatogastroenterology. 2003;50:1115-1118.

6. Yang CC, Chen CY, Lin XZ, Chang TT, Shin JS, Lin CY. Pyogenic liver abscess in Taiwan: emphasis on gas-forming liver abscess in diabetics. Am J Gastroenterol. 1993;88:1911-1915.

7. Ho MP, Chou AH, Cheung WK. Gas-forming pyogenic liver abscess in an elderly adult with diabetes mellitus. J Am Geriatr Soc. 2013;61:837-838.

8. Zhang Y, Zang GQ, Tang ZH, Yu YS. Fatal gas-forming pyogenic liver abscess due to Klebsiella pneumoniae. Rev Inst Med Trop Sao Paulo. 2013;55:144.

9. Yu WL, Ko WC, Cheng KC, Lee CC, Lai CC, Chuang YC. Comparison of prevalence of virulence factors for Klebsiella pneumoniae liver abscesses between isolates with capsular K1/K2 and non-K1/K2 serotypes. Diagn Microbiol Infect Dis. 2008;62:1-6.

10. Luo Y, Wang Y, Ye L, Yang J. Molecular epidemiology and virulence factors of pyogenic liver abscess causing Klebsiella pneumoniae in China. Clin Microbiol Infect. 2014;20:0818-24.

11. $\mathrm{Ng} \mathrm{H}$, Lam SM, Shum HP, Yan WW. Clostridium perfringens liver abscess with massive haemolysis. Hong Kong Med J. 2010;16:310-312.

12. Law ST, Lee MK. A middle-aged lady with a pyogenic liver abscess caused by Clostridium perfringens. World J Hepatol. 2012;4:252-255. 
13. Shiba H, Aoki H, Misawa T, Kobayashi S, Saito R, Yanaga K. Pneumoperitoneum caused by ruptured gas-containing liver abscess. J Hepatobiliary Pancreat Surg. 2007;14:210-211.

14. Siu LK, Yeh KM, Lin JC, Fung CP, Chang FY. Klebsiella pneumoniae liver abscess: a new invasive syndrome. Lancet Infect Dis. 2012;12:881-887.

15. Simon TG, Bradley J, Jones A, Carino G. Massive intravascular hemolysis from Clostridium perfringens septicemia: a review. J Intensive Care Med. 2014;29:327-33.

16. Tabarelli W, Bonatti H, Cejna M, Hartmann G, Stelzmueller I, Wenzl E. Clostridium perfringens liver abscess after pancreatic resection. Surg Infect (Larchmt). 2009;10:159-162.

17. Meyns E, Vermeersch N, Ilsen B, Hoste W, Delooz H, Hubloue I. Spontaneous intrahepatic gas gangrene and fatal septic shock. Acta Chir Belg. 2009;109:400-404.

18. Merino A, Pereira A, Castro P. Massive intravascular haemolysis during Clostridium perfrigens sepsis of hepatic origin. Eur J Haematol. 2010;84:278-279.

19. Rajendran G, Bothma P, Brodbeck A. Intravascular haemolysis and septicaemia due to Clostridium perfringens liver abscess. Anaesth Intensive Care. 2010;38:942-945.

20. van Bunderen CC, Bomers MK, Wesdorp E, Peerbooms P, Veenstra J. Clostridium perfringens septicaemia with massive intravascular haemolysis: a case report and review of the literature. Neth $J$ Med. 2010;68:343-346.

21. Kitterer D, Braun N, Jehs MC, Schulte B, Alscher MD, Latus J. Gas gangrene caused by clostridium perfringens involving the liver, spleen, and heart in a man 20 years after an orthotopic liver transplant: a case report. Exp Clin Transplant. 2014;12:165-168.

22. Qandeel H, Abudeeb H, Hammad A, Ray C, Sajid M, Mahmud S. Clostridium perfringens sepsis and liver abscess following laparoscopic cholecystectomy. J Surg Case Rep. 2012;2012:5.

23. Imai J, Ichikawa H, Tobita K, Watanabe N. Liver abscess caused by Clostridium perfringens. Intern Med. 2014;53:917-918.

24. Kurasawa M, Nishikido T, Koike J, Tominaga S, Tamemoto H. Gas-forming liver abscess associated with rapid hemolysis in a diabetic patient. World J Diabetes. 2014;5:224-229.

25. Rives C, Chaudhari D, Swenson J, Reddy C, Young M. Clostridium perfringens liver abscess complicated by bacteremia. Endoscopy. 2015;47 Suppl 1 UCTN: E457.

26. Eltawansy SA, Merchant C, Atluri P, Dwivedi S. Multi-organ failure secondary to a Clostridium perfringens gaseous liver abscess following a self-limited episode of acute gastroenteritis. $A m \mathrm{~J}$ Case Rep. 2015;16:182-186.

27. Khan MS, Ishaq MK, Jones KR. Gas-Forming Pyogenic Liver Abscess with Septic Shock. Case Rep Crit Care. 2015;2015:632873.

28. Li JH, Yao RR, Shen HJ, et al. Clostridium perfringens infection after transarterial chemoembolization for large hepatocellular carcinoma. World J Gastroenterol. 2015;21:4397-401.

29. Lim AG, Rudd KE, Halliday M, Hess JR. Hepatic abscess-associated Clostridial bacteraemia presenting with intravascular haemolysis and severe hypertension. BMJ Case Rep. 2016;2016. pii: 
bcr2015213253.

30. Meeuwes FO, Hukshorn CJ, Bloembergen P. Severe abdominal pain three weeks after a hemihepatectomy. Neth J Med. 2015;73:392-393.

31. García Carretero R, Romero Brugera M, Vazquez-Gomez O, Rebollo-Aparicio N. Massive haemolysis, gas-forming liver abscess and sepsis due to Clostridium perfringens bacteraemia. BMJ Case Rep. 2016;2016. pii: bcr2016218014.

32. Lee S. Kyanga, Thamer A. Bin Traikib, Nayef A. Alzahranic,d, David L. MorriseaSt. Microwave ablation of liver metastasis complicated by Clostridium perfringens gas-forming pyogenic liver abscess (GPLA) in a patient with past gastrectomy. Int J Surg Case Rep. 2016;27:32-35.

33. Hashiba M, Tomino A, Takenaka N, et al. Clostridium Perfringens Infection in a Febrile Patient with Severe Hemolytic Anemia. Am J Case Rep. 2016;17:219-223.

34. Paasch C, Wilczek S, Strik MW. Liver abscess and sepsis caused by Clostridium perfringens and Klebsiella oxytoca. Int J Surg Case Rep. 2017;41:180-183.

35. Yoshikawa T, Ohana M, Fukuda A. High Fever After Radiofrequency Ablation of Hepatocellular Carcinoma. Gastroenterology. 2018;155:e3-e4.

36. Shibazaki S, Yasumoto T, Nakaizumi T. Massive intravascular haemolysis due to Clostridium perfringens. BMJ Case Rep. 2018;2018. pii: bcr-2017-223464.

37. Hamada K, Sasaki Y. Gas-filled Cavities in the Liver. Intern Med. 2018;57:2277.

38. Tatsuta $T$, Wada $T$, Chinda $D$, et al. A case of gas-forming liver abscess with diabetes mellitus. Intern Med. 2011;50:2329-2332.

39. Oh JH, Jung SH, Jeon EJ. Gas-forming pyogenic liver abscess suspected on a plain chest X-ray. Korean J Intern Med. 2011;26:364.

40. Hagiya $H$, Kuroe $Y$, Nojima $H$, et al. Emphysematous liver abscesses complicated by septic pulmonary emboli in patients with diabetes: two cases. Intern Med. 2013;52:141-145.

41. Ho MP, Chou AH, Cheung WK. Gas-forming pyogenic liver abscess in an elderly adult with diabetes mellitus. J Am Geriatr Soc. 2013;61:837-8.

42. Paterson T, Inglis T. Images in clinical medicine. Metastatic gas. N Eng/ J Med. 2013;369:e33.

43. Keng LT, Fu YH, Lin YH. Gas-forming liver abscess with pneumocardia. J Emerg Med. 2014;46:e185186.

44. Kotwal A, Abraham K, Garcia M, Thompson M. Gas-Forming Pyogenic Liver Abscess in a Splenectomized Adult with Diabetes. J Diabetes Metab. 2005;6(8).

45. Chiou YW, Lin YT. Gas-forming Klebsiella pneumoniae liver abscess in a patient without diabetes. $J$ Microbiol Immunol Infect. 2015;48:709-710.

46. Takano Y, Hayashi M, Niiya F, et al. Life-threatening emphysematous liver abscess associated with poorly controlled diabetes mellitus: a case report. BMC Res Notes. 2017;10:117.

47. Kim YK, Jo KM, Jang JH, et al. Rapidly Fatal Emphysematous Osteomyelitis with Multiple Septic Emboli and Liver Abscess Caused by Klebsiella pneumoniae. Infect Chemother. 2018;50:268-273. 
48. Thng CB, Tan YP, Shelat VG. Gas-forming pyogenic liver abscess: A world review. Ann Hepatobiliary Pancreat Surg. 2018;22:11-18.

49. Lau YJ, Hu BS, Wu WL, Lin YH, Chang HY, Shi ZY. Identification of a major cluster of Klebsiella pneumoniae isolates from patients with liver abscess in Taiwan. J Clin Microbiol. 2000;38:412-414.

50. Okano H, Shiraki K, Inoue H, et al. Clinicopathological analysis of liver abscess in Japan. Int J Mol Med. 2002;10:627-630.

51. Chung DR, Lee SS, Lee HR, et al. Emerging invasive liver abscess caused by K1 serotype Klebsiella pneumoniae in Korea. J Infect. 2007;54:578-583.

52. Sartelli M, Abu-Zidan FM, Catena F, et al. Global validation of the WSES Sepsis Severity Score for patients with complicated intra-abdominal infections: a prospective multicentre study (WISS Study). World J Emerg Surg. 2015;10:61.

53. Chou FF, Sheen-Chen SM, Chen YS, Lee TY. The comparison of clinical course and results of treatment between gas-forming and non-gas-forming pyogenic liver abscess. Arch Surg. 1995;130:401-405.

54. Tan EY, Lee CW, Look Chee Meng M. Spontaneous pneumoperitoneum resulting from the rupture of a gas-forming pyogenic liver abscess. ANZ J Surg. 2005; 75:251-252.

55. Lee $\mathrm{CH}$, Chen IL, Chuah SK, et al. Impact of glycemic control on capsular polysaccharide biosynthesis and opsonophagocytosis of Klebsiella pneumoniae: Implications for invasive syndrome in patients with diabetes mellitus. Virulence. 2016;7:770-778.

56. Pan YJ, Fang HC, Yang HC, et al. Capsular polysaccharide synthesis regions in Klebsiella pneumoniae serotype K57 and a new capsular serotype. J Clin Microbiol. 2008;46:2231-2240.

57. Lee CH, Su LH, Liu JW, et al. Aspirin enhances opsonophagocytosis and is associated to a lower risk for Klebsiella pneumoniae invasive syndrome. BMC Infect Dis. 2014;14:47.

58. Li B, Zhao Y, Liu C, Chen Z, Zhou D. Molecular pathogenesis of Klebsiella pneumoniae. Future Microbiol. 2014;9:1071-1081.

59. Hatheway CL. Toxigenic clostridia. Clin Microbiol Rev. 1990;3:66-98.

60. Sakurai J, Nagahama M, Oda M. Clostridium perfringens alpha-toxin: characterization and mode of action. J Biochem. 2004;136:569-574.

61. Oda M, Kabura M, Takagishi T, et al. Clostridium perfringens alpha-toxin recognizes the GM1a-TrkA complex. J Biol Chem. 2012;287:33070-33079.

62. Jung J, Park KH, Park SY, et al. Comparison of the clinical characteristics and outcomes of Klebsiella pneumoniae and Streptococcus pneumoniae meningitis. Diagn Microbiol Infect Dis. 2015;82:87-91.

\section{Tables}


Table 1 Cases of K. pneumoniae GFPLA published from January 2009 to March 2019

\begin{tabular}{|c|c|c|c|c|c|c|c|c|c|c|c|c|c|}
\hline Author & Year & $\begin{array}{l}\text { Age } \\
(\mathrm{yr})\end{array}$ & Sex & Country & Condition(s) & Klebsiella pneumoniae originates & Diagnose & $\begin{array}{l}\text { Abscess } \\
\text { location }\end{array}$ & $\begin{array}{c}\text { Abscess } \\
\text { diameter }(\mathrm{cm})\end{array}$ & $\begin{array}{c}\text { Focus } \\
\text { removed }\end{array}$ & HML & SSE & TTD \\
\hline Tatsuta et al $^{[38]}$ & 2011 & 43 & $\mathrm{M}$ & Japan & DM & Blood and pus culture & CT, XR, US & Right & 13 & Yes & No & - & - \\
\hline Oh et $\mathrm{al}^{[39]}$ & 2011 & 70 & M & Korea & $\mathrm{DM}$ & Pus cultures & CT & Right & - & Yes & No & - & - \\
\hline Hagiya et al ${ }^{[40]}$ & 2012 & 58 & M & Japan & $\mathrm{DM}$ & Blood and pus culture & CT & Right & - & Yes & No & - & - \\
\hline Hagiya et al ${ }^{[40]}$ & 2012 & 61 & $\mathrm{~F}$ & Japan & $\mathrm{DM}$ & Blood cultures & CT & Right & - & Yes & No & - & - \\
\hline Ho et al ${ }^{[41]}$ & 2013 & 66 & $\mathrm{~F}$ & Taiwan & $\mathrm{DM}$ & Blood cultures & CT & Right & - & Yes & No & - & - \\
\hline Zhang et $\mathrm{al}^{[8]}$ & 2013 & 76 & $\mathrm{~F}$ & China & $\mathrm{DM}$ & Blood and pus culture & CT & Right & 14 & Yes & No & No & $5 \mathrm{~d}$ \\
\hline Paterson et al ${ }^{[42]}$ & 2013 & 60 & M & Australia & None & Blood and pus culture & CT & Right & - & Yes & No & - & - \\
\hline Keng et $\mathrm{al}^{[43]}$ & 2014 & 76 & M & Taiwan & $\mathrm{DM}$ & Blood cultures & CT & Right & - & Yes & No & - & - \\
\hline Kotwal et al ${ }^{[44]}$ & 2015 & 75 & $\mathrm{~F}$ & USA & $\mathrm{DM}$ & Blood cultures & CT, MRCP & Right & 6 & Yes & No & - & - \\
\hline Chiou et al ${ }^{[45]}$ & 2015 & 72 & M & Taiwan & None & Blood and pus culture & CT & Left & 8 & Yes & No & - & - \\
\hline Yuichi et al ${ }^{[46]}$ & 2017 & 69 & M & Japan & $\mathrm{DM}$ & Blood and pus culture & CT, US & Right & 5 & Yes & No & - & - \\
\hline Kim et al ${ }^{[47]}$ & 2017 & 79 & $\mathrm{~F}$ & Korea & DM, hypertension & Blood cultures & CT & Right & 3 & Yes & No & No & $19 \mathrm{~h}$ \\
\hline
\end{tabular}

CT: Computed tomography; XR:X-Ray; US: Ultrasound scan; MRCP: Magnetic resonance cholangiopancreatography; HML: Hemolysis; SSE: Survival of septic episode; TTD: Time to death

Table 2 Cases of C. perfringens GFPLA published from January 2009 to March 2019

\begin{tabular}{|c|c|c|c|c|c|c|c|c|c|c|c|c|c|}
\hline Author & Year & & & Country & Condition(s) & Originates & Diagnose & $\begin{array}{l}\text { Abscess } \\
\text { location } \\
\end{array}$ & $\begin{array}{l}\text { Abscess } \\
\text { size }(\mathrm{cm})\end{array}$ & $\begin{array}{c}\text { Focus } \\
\text { removed }\end{array}$ & HML & SSE & TTD \\
\hline Tabarelli et al ${ }^{[16]}$ & 2009 & 65 & $\mathrm{~F}$ & Austria & Pancreatic cancer & Blood culture & $\mathrm{CT}$ & Right & 9 & No & No & No & $27 \mathrm{~d}$ \\
\hline Meyns et al ${ }^{[17]}$ & 2009 & 64 & M & Belgium & DM & Bbod and pus culture & $\mathrm{CT}, \mathrm{XR}$ & Right & - & No & Yes & No & $\mathrm{N} / \mathrm{A}^{1}$ \\
\hline Merino et al ${ }^{[18]}$ & 2009 & 83 & $\mathrm{~F}$ & Spain & Cholangitis & Blood culture & $\mathrm{CT}$ & N/A & - & No & Yes & No & $3 \mathrm{~d}$ \\
\hline Ng et al ${ }^{[11]}$ & 2010 & 61 & $\mathrm{~F}$ & Hong Kong & DM & Blood culture & CT & Left & - & Yes & Yes & Yes & - \\
\hline Rajendran et al ${ }^{[19]}$ & 2010 & 58 & M & UK & None & Blood culture & CT, XR & Right & - & Yes & No & Yes & - \\
\hline Bunderen et al ${ }^{[20]}$ & 2010 & 74 & M & Netherlands & Cholangitis & Blood culture & $\mathrm{NA}^{1}$ & N/A & - & No & Yes & Yes & - \\
\hline Kitterer et al ${ }^{[21]}$ & 2012 & 71 & M & Germany & Fiver transplant & Blood culture & CT & Right & 8 & Yes & No & No & $6 \mathrm{~h}$ \\
\hline Qandeel et al ${ }^{[22]}$ & 2012 & 59 & M & UK & DM;ERCP & Blood culture & $\mathrm{CT}$ & Right & - & Yes & Yes & Yes & - \\
\hline Law et al ${ }^{[12]}$ & 2012 & 50 & $\mathrm{~F}$ & Hong Kong & Rectal cancer & Blood culture & $\mathrm{CT}$ & Right & 15 & No & No & No & $7 d$ \\
\hline Imai et al ${ }^{[23]}$ & 2014 & 76 & M & Japan & None & Bbod and pus culture & $\mathrm{CT}, \mathrm{XR}$ & Right & 65 & No & Yes & No & $6 \mathrm{~h}$ \\
\hline Kurasawa et al ${ }^{[24]}$ & 2014 & 65 & M & Japan & DM & Blood culture & CT & Right & - & No & No & No & $6 \mathrm{~h}$ \\
\hline Rives et al ${ }^{[25]}$ & 2015 & 63 & $\mathrm{M}$ & USA & Colon cancer,ERCP & Blood culture & CT & Left & 3.1 & No & No & Yes & - \\
\hline Eltawansy et al ${ }^{[26]}$ & 2015 & 81 & $\mathrm{~F}$ & USA & DM, Gastroenteritis & Blood culture & CT & Right & - & No & No & Yes & - \\
\hline Khan et al ${ }^{[27]}$ & 2015 & 77 & M & USA & DM & Blood culture & CT, XR & Left & - & No & Yes & No & $9 \mathrm{~h}$ \\
\hline Liet al ${ }^{[28]}$ & 2015 & 71 & M & China & Cholangtis & Blood culture & $\mathrm{CT}$ & Right & - & No & Yes & Yes & - \\
\hline Lim et $\mathrm{al}^{[29]}$ & 2015 & 58 & M & USA & None & Blood culture & CT & Both & 5 & No & Yes & No & $8 \mathrm{~h}$ \\
\hline Meeuwes et al ${ }^{[30]}$ & 2015 & 71 & $\mathrm{~F}$ & Netherlands & Hemi-hepatectomy;ERCP & Blood culture & CT & Right & - & No & Yes & Yes & - \\
\hline García et al ${ }^{[31]}$ & 2016 & 65 & M & Spain & DM & Blood culture & CT & Right & 4.8 & No & Yes & Yes & - \\
\hline Kyang et al ${ }^{[32]}$ & 2016 & 84 & M & Australia & Gastric cancer & Pus culture & $\mathrm{CT}$ & Right & - & No & No & Yes & - \\
\hline Hashba et al ${ }^{[33]}$ & 2016 & 82 & M & Japan & DM & Blood culture & CT & Left & - & No & Yes & No & $2 \mathrm{~h}$ \\
\hline Paasch et al ${ }^{[34]}$ & 2017 & 65 & M & Germany & DM & Pus culture & $\mathrm{CT}$ & Right & 7 & Yes & No & Yes & - \\
\hline Yoshikawa et al ${ }^{[35]}$ & 2017 & 70 & M & Japan & Hepatocellular carcinoma;TACE & Pus culture & CT & Right & - & No & No & Yes & - \\
\hline Shibazaki et al ${ }^{[36]}$ & 2018 & 68 & $\mathrm{~F}$ & Japan & DM & Blood culture & CT & Left & - & No & No & No & $1 \mathrm{~h}$ \\
\hline Hamada et al ${ }^{[37]}$ & 2018 & 68 & M & Japan & Gastric cancer & Blood culture & CT & Right & - & No & Yes & No & $10 \mathrm{~h}$ \\
\hline
\end{tabular}

XR: X-Ray; 1Exact time of TTD was not discussed; HML: Hemolysi; SSE: Survival of septic episode; TTD: Time to death 
Table 3 laboratory examination of K. pneumoniae GFPLA

\begin{tabular}{ccccc}
\hline Ref. & $\begin{array}{c}\text { HbA1C } \\
(\%)\end{array}$ & $\begin{array}{c}\text { AST } \\
(\mathrm{IU} / \mathrm{L})\end{array}$ & $\begin{array}{c}\text { ALT } \\
(\mathrm{IU} / \mathrm{L})\end{array}$ & $\begin{array}{c}\text { ALP } \\
(\mathrm{IU} / \mathrm{L})\end{array}$ \\
\hline 38 & 14.3 & 149 & 171 & 602 \\
39 & - & - & - & - \\
40 & 11.4 & 164 & 145 & 1865 \\
40 & 10.5 & 116 & 142 & 880 \\
41 & - & 260 & - & - \\
8 & - & - & - & - \\
42 & - & - & - & - \\
43 & - & 379 & 237 & - \\
44 & 8.2 & 220 & 125 & 354 \\
45 & 6.2 & 206 & 248 & 300 \\
46 & 14.6 & 371 & 331 & 675 \\
47 & 8.2 & - & - & - \\
\hline
\end{tabular}


Table 4 laboratory examination of $C$. perfringens GFPLA

\begin{tabular}{|c|c|c|c|c|c|c|}
\hline Ref. & $\begin{array}{c}\mathrm{Hb} \\
(\mathrm{g} / \mathrm{dL})\end{array}$ & ALT & AST & ALP & $\begin{array}{l}\text { Bilirubin } \\
\text { (mg/dl) }\end{array}$ & $\begin{array}{c}\mathrm{LDH} \\
(\mathrm{IU} / \mathrm{L})\end{array}$ \\
\hline 16 & - & - & - & - & - & - \\
\hline 17 & 7.2 & 84 & 139 & - & 8.27 & 980 \\
\hline 18 & 6 & - & - & - & 19.6 & 2288 \\
\hline 11 & 14.3 & 1314 & - & 112 & - & 4054 \\
\hline 19 & 9 & - & - & - & - & - \\
\hline 20 & 9.8 & 261 & 419 & 271 & 23.7 & 2300 \\
\hline 21 & 11.2 & - & - & - & 3.7 & - \\
\hline 22 & 6.8 & - & - & - & - & - \\
\hline 12 & 8.3 & - & - & 302 & 8.95 & 1132 \\
\hline 23 & 12.2 & - & 1388 & & 10.5 & 4767 \\
\hline 24 & 13.5 & 261 & 297 & 469 & 6.4 & 6203 \\
\hline 25 & - & & & & 7.6 & - \\
\hline 26 & - & 231 & 318 & 494 & 0.42 & - \\
\hline 27 & 6 & 370 & 1179 & 238 & 9.5 & 3442 \\
\hline 28 & 11.8 & 1652 & 787 & - & 18.89 & - \\
\hline 29 & 12.4 & - & - & - & - & - \\
\hline 30 & - & - & - & - & 10.47 & - \\
\hline 31 & 14.6 & 402 & - & - & - & - \\
\hline 32 & - & 825 & 894 & - & - & - \\
\hline 33 & 8.3 & & 1366 & - & 9 & 10321 \\
\hline 34 & - & - & - & - & - & - \\
\hline 35 & - & - & - & - & - & - \\
\hline 36 & 6.9 & 690 & 1905 & - & 8.1 & - \\
\hline 37 & - & - & - & - & - & - \\
\hline
\end{tabular}

\section{Supplementary Files}

This is a list of supplementary files associated with this preprint. Click to download.

- PRISMA2009checklist.doc 